\title{
Statins prevent adverse effects of postnatal glucocorticoid therapy on the developing brain in rats
}

\author{
Deodata Tijsseling', Emily J. Camm², Hans G. Richter², Emilio A. Herrera², Andrew D. Kane², Youguo Niu², Christine M. Cross²,
} Willem B. de Vries ${ }^{1}$, Jan B. Derks ${ }^{1}$ and Dino A. Giussani ${ }^{2}$

BACKGROUND: Postnatal glucocorticoid therapy in the treatment of chronic lung disease benefits lung function, however it adversely affects brain development. We hypothesized that combined postnatal glucocorticoid and statin therapy diminishes adverse effects of glucocorticoids on the developing brain.

METHODS: On postnatal days (P) 1-3, one male pup per litter received i.p. injections of saline (control (C), $n=13$ ) or dexamethasone $(0.5,0.3,0.1 \mu \mathrm{g} / \mathrm{g} ; \mathrm{D}, n=13), \pm$ pravastatin (10 mg/ kg i.p.; $C P, n=12 ; D P, n=15)$. Statins or saline continued from P4-6. At P21, brains were perfusion fixed for histological and stereological analyses.

RESULTS: Relative to controls, dexamethasone reduced total $(837 \pm 23$ vs. $723 \pm 37)$, cortical ( $378 \pm 12$ vs. $329 \pm 15)$, and deep gray matter ( $329 \pm 12$ vs. $284 \pm 15)$ volume $\left(\mathrm{mm}^{3}\right)$, cortical neuronal number $\left(23 \pm 1\right.$ vs. $\left.19 \pm 1 \times 10^{6}\right)$, and hippocampal neuronal soma volume (CA1: 1,206 \pm 32 vs. $999 \pm 32$; dentate gyrus: $679 \pm 28$ vs. $542 \pm 24 \mu m^{3}$; all $\left.P<0.05\right)$. Dexamethasone increased the glial fibrillary acidic protein-positive astrocyte density in the white matter $\left(96 \pm 2\right.$ vs. $\left.110 \pm 4 / 0.1 \mathrm{~mm}^{2}\right) ; P<0.05$. These effects no longer occurred in brains from pups treated with combined dexamethasone and pravastatin. Pravastatin alone had no effect on these variables.

CONCLUSION: Concomitant dexamethasone with statins in premature infants may be safer for the developing brain than dexamethasone alone in the treatment of chronic lung disease.

hronic lung disease is a common outcome in extremely preterm neonates with severe respiratory distress syndrome and carries a high incidence of morbidity and mortality (1). Glucocorticoids, predominantly dexamethasone, are used to decrease inflammatory responses, accelerate surfactant production, and lung maturation, thereby improving respiratory function (2).

Despite the well-established beneficial effects of postnatal glucocorticoid therapy, there has been serious growing concern regarding their clinical use because of unwanted side effects on somatic growth and weight gain in premature babies $(3,4)$. Accumulating evidence from clinical trials has also demonstrated an association between postnatal steroid therapy and adverse neuromotor and cognitive outcomes $(4,5)$. Furthermore, experimental studies in neonatal animals have demonstrated adverse effects of potent glucocorticoids, such as dexamethasone, on brain growth, cell division, differentiation, myelination, apoptosis, and neurogenesis (6-8).

To date, the mechanism underlying the unwanted side effects of glucocorticoid therapy on the developing brain remains unknown, preventing the identification of plausible modified therapies to maintain the beneficial, but prevent the adverse, effects of steroid treatment. Glucocorticoids are known to decrease nitric oxide (NO) bioavailability and increases in NO via antioxidant treatment have been shown to prevent or partially restore glucocorticoid-induced cardiovascular dysfunction and hypertension $(9,10)$, implying impaired NO physiology as the culprit mechanism mediating unwanted side effects of glucocorticoids. Recent evidence indicates that statins, which are widely prescribed drugs for the primary prevention of coronary heart disease (11), have additional beneficial actions beyond that of cholesterol reduction via increasing NO bioavailability. Indeed, clinical and experimental evidence indicates that these pleiotropic effects of statins, by improving endothelial function, might be potentially useful for the treatment of neurologic disorders, such as ischemic stroke, Parkinson's disease, Alzheimer's disease, and vascular dementia (12-14). However, whether statins can protect the developing brain has never been investigated. Using an established experimental model of prematurity and postnatal glucocorticoid therapy $(10,15,16)$, this study tested in rats the hypothesis that combined postnatal glucocorticoid and statin therapy will diminish the adverse effects of glucocorticoids on the developing brain by preserving NO bioavailability.

\section{RESULTS}

\section{Body and Brain Weights}

Compared with control offspring, dexamethasone significantly reduced body and brain weight at postnatal day 21 (P21), whether expressed as absolute weight or as a percentage of control weight (Figure 1a-d; $P<0.05$ ). Postnatal treatment with combined dexamethasone and pravastatin restored brain weight to control levels, however body weight remained 
a

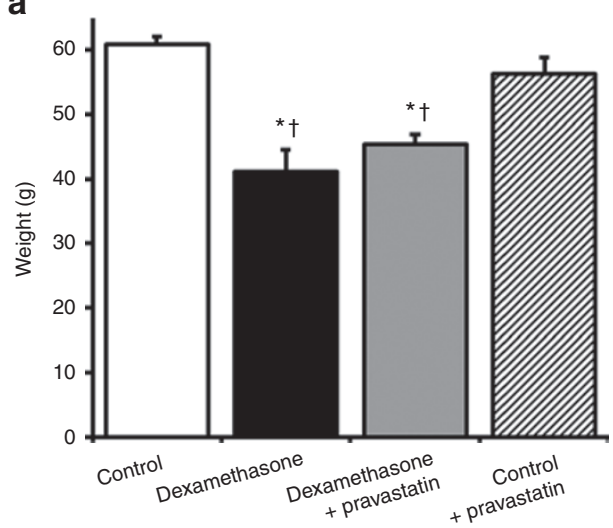

C

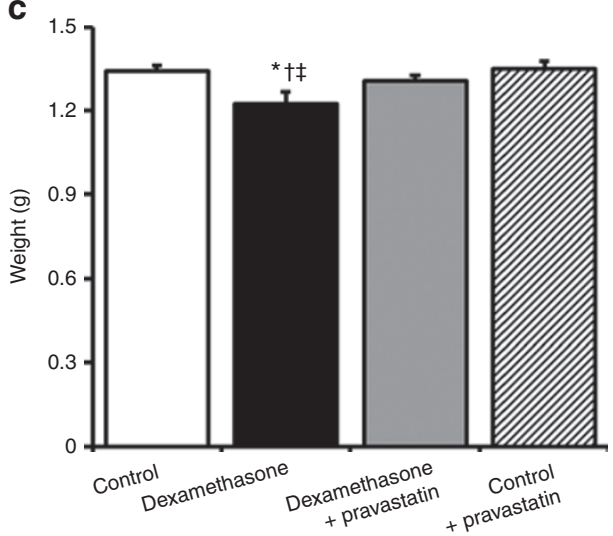

b

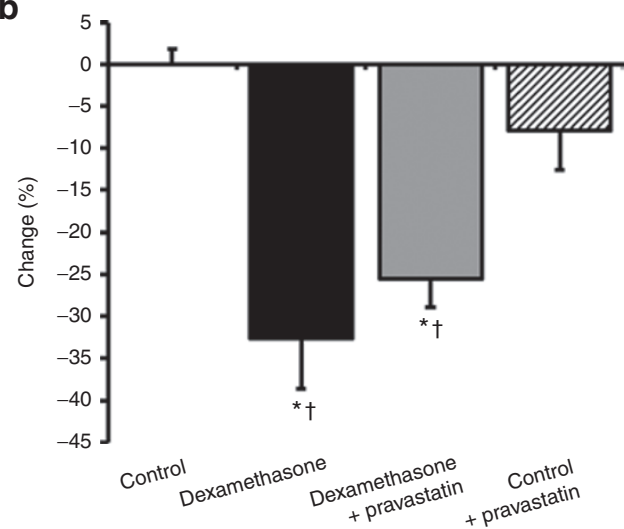

d

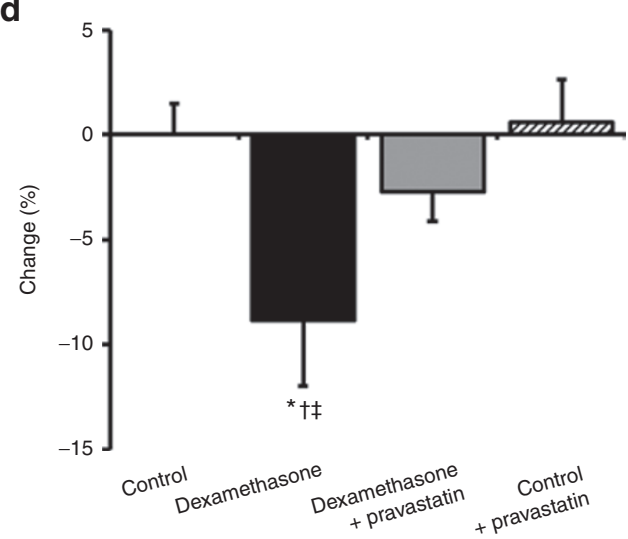

Figure 1. Effects on body and brain weight. (a) Total body weight, (b) percentage change from control body weight, (c) total brain weight, (d) percentage change from control brain weight at postnatal day 21 in control $(n=8)$, dexamethasone $(n=7)$, dexamethasone with pravastatin $(n=8)$, and control with pravastatin $(n=7)$ pups. ${ }^{*} P<0.05$ vs. control; ${ }^{\dagger} P<0.05$ vs. control with pravastatin; ${ }^{\ddagger} P<0.05$ vs. dexamethasone with pravastatin (One-way ANOVA + Student-Newman-Keuls).

reduced. Postnatal treatment of control animals with pravastatin alone had no effect on body or brain weight (Figure 1a-d).

\section{Cerebral Histological Analysis}

Gross morphology and regional brain volumes. At P21, examination of the cresyl violet-stained coronal sections did not reveal any gross alterations in cytoarchitecture, cellular morphology, or anatomical organization of the cortex or hippocampus between the four experimental groups. However, relative to control animals, postnatal treatment with dexamethasone reduced absolute cerebral volume; this decrease appeared to be due to significant reductions in cortical and deep gray matter volumes (Figure 2a; $P<0.05)$. Concomitant postnatal treatment with pravastatin restored total cerebral, cortical, and deep gray matter volumes to control levels. No significant alterations in hippocampal volumes or white matter volumes were observed between the groups. Postnatal treatment with pravastatin alone had no effect on absolute or regional brain volumes (Figure 2a). Postnatal dexamethasone treatment reduced brain volume proportionally as differences disappeared when comparing compartmental volumes expressed relative to total cerebral volume (Figure $2 \mathbf{b}$ ).

Neuronal numbers and soma volumes. Compared with control offspring, neuronal number in the cortex was significantly reduced in P21 offspring following postnatal treatment with dexamethasone $(P<0.05)$; soma volume of cortical neurons was unaltered (Table 1). In contrast, in the hippocampus, neuronal numbers were unaltered; however, soma volumes were significantly reduced in the CA1 and the dentate gyrus of P21 pups treated with dexamethasone postnatally (Table 2; $P<$ $0.05)$. These alterations in neuronal number and soma volume no longer occurred in P21 pups treated with combined dexamethasone and pravastatin postnatally. Postnatal treatment with pravastatin alone had no effect on neuronal number or soma volume.

Astrocyte density. Postnatal treatment with dexamethasone significantly increased glial fibrillary acidic protein (GFAP)-positive astrocyte density in the cingulate white matter (Figure 3; $P<0.05)$. Postnatal concomitant treatment of dexamethasone with pravastatin normalized GFAP-positive astrocyte density to control levels. Postnatal treatment with pravastatin alone had no effect on GFAP-positive astrocyte density (Figure 3).

Volume fraction of blood vessels and myelination. No differences in the volume fraction of blood vessels in the primary and secondary motor cortex, the primary somatosensory cortex, lateral and medial parietal association cortex, the primary and 
secondary visual cortex (control "C": $9.0 \pm 0.7 \%$; dexamethasone "D": $8.4 \pm 0.3 \%$; dexamethasone with pravastatin "DP": $7.2 \pm 0.2 \%$; control with pravastatin "CP": $7.9 \pm 0.9 \%$; $P>0.05$ ) or the stratum lacunosum-moleculare and stratum radiatum of the hippocampus (C: $6.2 \pm 0.3 \%$; D: $7.0 \pm 0.5 \%$; DP: $6.0 \pm 0.4 \%$; $\mathrm{CP}: 6.5 \pm 0.4 \%$; $P>0.05)$ were found among the four experimental groups. Similarly, no differences were found among the groups in relation to the optical density of myelin basic protein-stained fibers in the cortex (C: $0.39 \pm 0.03$; D: $0.38 \pm 0.02$; DP: $0.44 \pm 0.02$; CP: $0.39 \pm 0.01 ; P>0.05)$ or the extent of myelination in the cortex (C: $73.1 \pm 0.6 \%$; D: $70.0 \pm 1.4 \%$; DP:
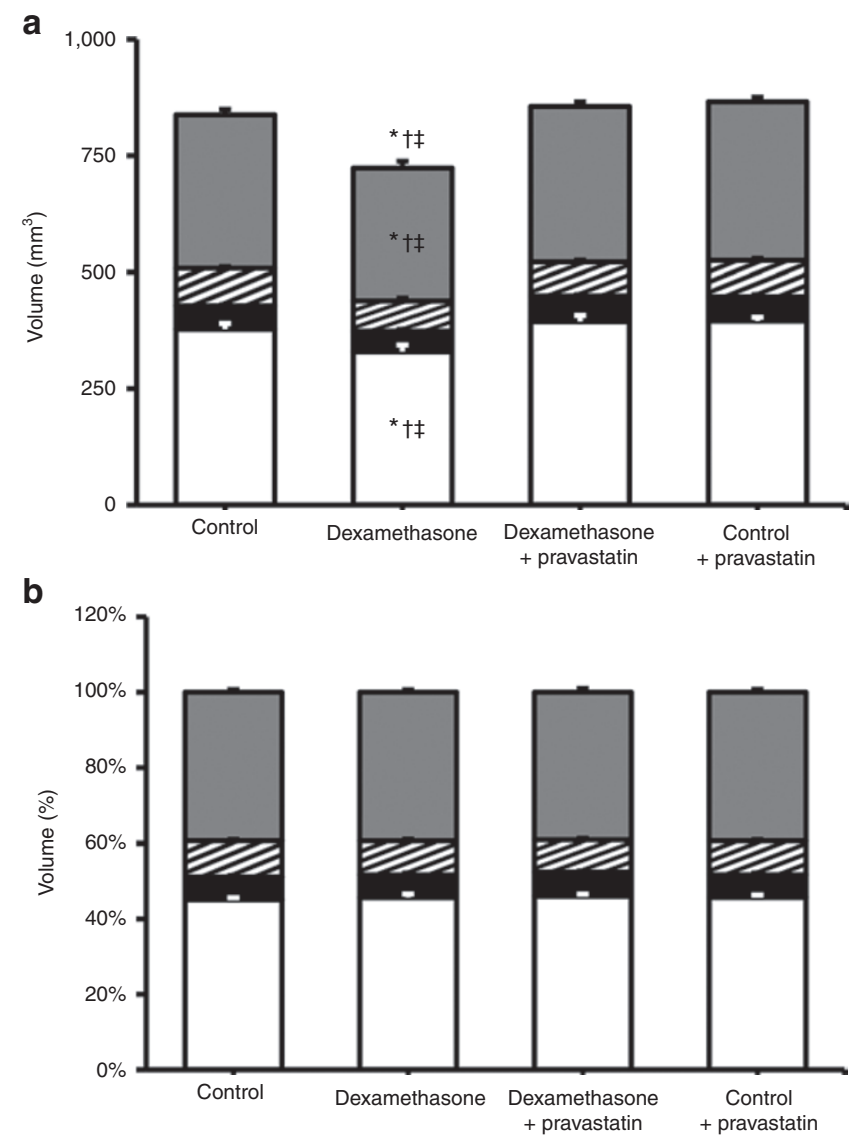

Figure 2. Effects on brain volume. (a) Total brain volume and volumes of deep gray matter, hippocampus, white matter, and cortex (b) deep gray matter volume, hippocampal volume, white matter volume, and cortical volume as a percentage of total brain volume, at postnatal day 21 in control $(n=8)$, dexamethasone $(n=7)$, dexamethasone with pravastatin $(n=8)$, and control with pravastatin $(n=7)$ pups. Gray bar: deep gray matter; striped bar: hippocampus; black bar: white matter; white bar: cortex. ${ }^{*} P<0.05$ vs. control; ${ }^{\dagger} P<0.05$ vs. control with pravastatin; ${ }^{\ddagger} P<0.05$ vs. dexamethasone with pravastatin (One-way ANOVA + Student-Newman-Keuls).
$70.9 \pm 0.7 \%$; CP: $72.4 \pm 0.8 \% ; P>0.05)$. However, the optical density of myelin basic protein-stained fibers was significantly increased in the corpus callosum of P21 offspring treated postnatally with combined dexamethasone and pravastatin, but not pravastatin alone, when compared with control offspring (C: $0.49 \pm 0.04$; D: $0.46 \pm 0.03$; DP: $0.59 \pm 0.04$; CP: $0.46 \pm 0.01$; $P<0.05)$.

\section{NO Bioavailability}

Compared with controls, postnatal treatment with dexamethasone led to a significant decrease in plasma $\mathrm{NOx}$, comprising plasma nitrite $\left(\mathrm{NO}_{2}^{-}\right)$and nitrate $\left(\mathrm{NO}_{3}^{-}\right)(22.3 \pm 2.2 \mu \mathrm{mol} / \mathrm{l} \mathrm{vs}$. $13.1 \pm 0.8 \mu \mathrm{mol} / \mathrm{l} ; P<0.05)$ at $\mathrm{P} 21$. In contrast, this decrease did not occur in pups following combined postnatal dexamethasone and pravastatin treatment $(19.9 \pm 4.4 \mu \mathrm{mol} / \mathrm{l} ; P<$ $0.05)$. Postnatal pravastatin treatment alone also significantly reduced plasma NOx levels $(11.6 \pm 2.1 \mu \mathrm{mol} / \mathrm{l} ; P<0.05)$.

\section{DISCUSSION}

The postnatal rat is an established experimental model of human prematurity, as postnatal development of neuronal, cardiovascular, and respiratory function in this species compares with prenatal milestones in the human $(17,18)$. Data in the present study show that postnatal treatment of rat pups with dexamethasone in human clinically relevant doses decreased regional brain volumes, cortical neuronal number and hippocampal soma volume, while increasing the density of GFAPpositive astrocytes in the white matter when measured at weaning. Postnatal treatment with dexamethasone also led to a fall in plasma NOx concentrations at weaning. Furthermore, the data show that postnatal combined treatment of dexamethasone with statins prevented the fall in plasma NOx concentrations and the adverse effects on cerebral development at P21. Treatment of newborn pups with pravastatin alone had no adverse effects on brain development, but it reduced plasma NOx concentrations at weaning.

Stereological analysis of brain tissue in the present study revealed that the volumes of the cerebrum, cortex, and deep gray matter were significantly decreased in dexamethasonetreated offspring. Brains of dexamethasone-treated pups were proportionally smaller, as regional alterations were not observed when volumes were expressed as a percentage of total brain volume. The reduction in whole-cerebral tissue volume and regional volumes induced by dexamethasone could be a result of a reduction in neuronal number, and/ or soma volume and/or a decrease or delay in dendritic outgrowth. To differentiate between some of these possibilities,

Table 1. Neuronal numbers and soma volumes in the cortex

\begin{tabular}{|c|c|c|c|c|}
\hline & C & D & DP & $\mathrm{CP}$ \\
\hline Cortical neuronal number $\left(\times 10^{6}\right)$ & $22.8 \pm 1.05$ & $18.9 \pm 0.78^{*}$ & $20.4 \pm 1.14$ & $20.7 \pm 0.87$ \\
\hline Average soma volume of cortical neurons $\left(\mu \mathrm{m}^{3}\right)$ & $1,176.4 \pm 21.1$ & $1,095.9 \pm 41.0$ & $1,140.7 \pm 43.9$ & $1,179.1 \pm 28.8$ \\
\hline
\end{tabular}




\section{Articles $\mid$ Tijsseling et al.}

Table 2. Neuronal numbers and soma volumes in the hippocampus

\begin{tabular}{|c|c|c|c|c|}
\hline & $\mathrm{C}$ & $\mathrm{D}$ & DP & $\mathrm{CP}$ \\
\hline Neuronal number in CA1 $(\times 1,000)$ & $408.6 \pm 16.5$ & $400.4 \pm 23.4$ & $415.1 \pm 23.2$ & $416.3 \pm 10.0$ \\
\hline Average soma volume of $C A 1$ neurons $\left(\mu \mathrm{m}^{3}\right)$ & $1,205.5 \pm 32.4$ & $999.3 \pm 32.3^{*+}$ & $1,109.4 \pm 54.8$ & $1,178.5 \pm 79.1$ \\
\hline Average soma volume of $C A 2 / 3$ neurons $\left(\mu \mathrm{m}^{3}\right)$ & $1,557.5 \pm 46.1$ & $1,381.8 \pm 93.5$ & $1,564.5 \pm 116.0$ & $1,575.5 \pm 66.2$ \\
\hline Neuronal number in dentate gyrus $(\times 1,000)$ & $990.6 \pm 52.1$ & $931.2 \pm 82.5$ & $1,070.0 \pm 48.6$ & $874.4 \pm 57.1$ \\
\hline
\end{tabular}

Neuronal numbers and soma volumes in the hippocampus of control $(C, n=8)$, dexamethasone $(D, n=7)$, dexamethasone with pravastatin (DP, $n=6)$, and control with pravastatin $(C P, n=6)$ pups.

${ }^{*} P<0.05$ vs. $C,{ }^{\dagger} P<0.05$ vs. CP (One-way ANOVA + Student-Newman-Keuls).
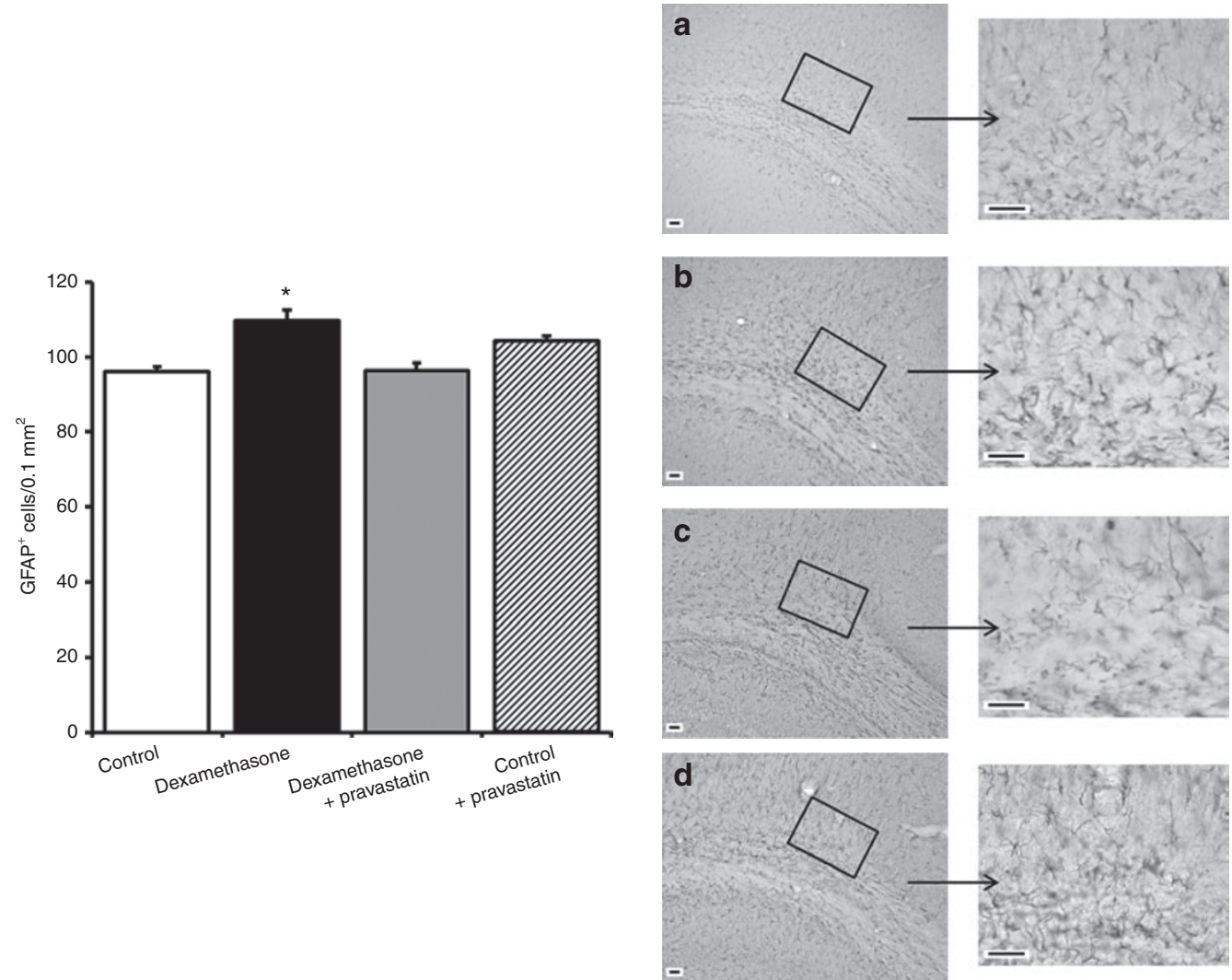

Figure 3. Density of GFAP-positive astrocytes in the cingulate white matter. Density of GFAP-positive astrocytes in control $(n=6)$, dexamethasone $(n=6)$, dexamethasone with pravastatin $(n=6)$, and control with pravastatin $(n=6)$ pups. ${ }^{*} P<0.05$ vs. control (One-way ANOVA + Student-Newman-Keuls). (a-d, left): photomicrographs show in low power the area of the cingulate white matter where the number of GFAP-positive astrocytes were counted. a: Control, b: dexamethasone, c: dexamethasone with pravastatin, $\mathbf{d}$ : control with pravastatin. (a-d, right): Photomicrographs show the boxed areas in high power. All scale bars represent $30 \mu \mathrm{m}$. GFAP, glial fibrillary acidic protein.

we determined total neuronal number and soma volume in the cortex and hippocampus. The data revealed that cortical neuronal number, but not soma volume, was reduced following dexamethasone treatment. However, the density of cortical neurons remained unaltered following dexamethasone treatment, indicating a reduction in neurons in proportion to the reduction in the size of the cortex. Dexamethasone-dependent reductions in cortical neuronal number have been previously described by Crochemore et al. (19) and may be due to a suppression of neurogenesis, as reported in tree shrews following psychosocial stress or following corticosterone treatment in rats $(20,21)$ or due to impaired neuronal proliferation (8) or enhanced cell death (22), as also reported in rats following dexamethasone treatment. Additional data in our study show that soma volume, but not neuronal number, was reduced in the CA1 and the dentate gyrus of the hippocampus following postnatal dexamethasone treatment. The observed reduction in neuronal soma volume may be related to a decrease in cellular function (23), or reflect a delay in neuronal differentiation and/or dendritic growth, as previously described in the fetal baboon brain (6). The decrease could further indicate a preferential loss of larger neurons, which has been observed in neurodegenerative diseases (24), or reflect neuronal shrinkage, which often precedes neurodegeneration (25). 
Beside the changes in gray matter, we were also interested in white matter changes because of the association of dexamethasone with increased rates of cerebral palsy (2). White matter damage is the leading pathological condition underlying cerebral palsy in children born prematurely and is characterized by an inflammatory response, astrogliosis, increased cell death and myelination impairment (26). Data in the present study show that postnatal dexamethasone therapy was also associated with a significant increase in the density of GFAP-positive astrocytes in the cingulate white matter.

Results in the present study not only confirm the deleterious effects of postnatal dexamethasone administration on the developing brain, as reported in several species, but they also extend these findings to show that combined administration of postnatal dexamethasone with pravastatin prevented the decrease in regional brain volumes, cortical neuronal number, and the reduction in hippocampal soma volume in the CA1 and the dentate gyrus. The group of 5-hydroxy-3-methylglutaryl-coenzyme A (HMG CoA) reductase inhibitors, or statins, has become one of the most effective and widely prescribed drugs for the primary and secondary prevention of cardiovascular disease (11). In addition to their established lipid-lowering actions, positive effects of statins have been demonstrated on the cardiovascular system (27); benefits which have all been credited to increases in NO bioavailability. To explore enhanced NO bioavailability as a potential mechanism mediating the protective effect of statins in dexamethasone-treated pups, we measured plasma concentrations of NOx in the weanling rats. Plasma nitrite and nitrate (NOx) are stable end products of NO, providing a good first-order index on enhanced NO bioavailability. Glucocorticoid excess has been reported to enhance reactive oxygen species (ROS) generation directly (9) or secondary to endothelial nitric oxide synthase (eNOS) uncoupling by limiting the availability of cofactors, such as L-arginine and tetrahydrobiopterin (BH4) (28). Oxygen free radicals, such as the superoxide anion, readily combine with NO, reducing its bioavailability (9). Reduced NO bioavailability promotes increased vascular resistance and thereby a decrease in perfusion, including a reduction in blood flow in the cerebral vascular bed (29). One mechanism by which statins may convey protection following dexamethasone treatment may therefore be by enhancing the bioavailability of NO to favor maintained perfusion, especially in highly NO-dependent circulations, such as the cerebral vascular bed. Statins have also been shown to promote phosphorylation of Akt (12). Phosphorylation increases protein kinase activity, leading to Akt-mediated phosphorylation of eNOS, which stimulates NO production (30). NO activates soluble guanylyl cyclase (sGC) leading to the formation of cGMP (12). In the central nervous system, NO/cGMP signaling promotes synaptic plasticity, neurogenesis, and angiogenesis $(31,32)$ providing an additional pathway via which statininduced increases in NO bioavailability may be protective on the developing brain.

Of note, data in the present study also show that treatment of postnatal rats with pravastatin alone did not have any adverse effects on any measured variable in the developing brain, but it did significantly reduce plasma NOx levels at P21. It is likely that the fall in plasma NOx following statin treatment alone is the result of negative feedback due to excess NO bioavailability and thereby having no adverse effects on the brain. Chronic increases in NO have been reported to down-regulate sGC expression (33) and to increase phosphodiesterase function (34), lowering the bioavailability of NO. This idea is consistent with dexamethasone-induced reductions in the concentration of the vasodilator gas with deleterious effects on the brain, vs. effects of statins in dexamethasone-treated pups replenishing appropriate NO concentrations and therefore, protecting the brain.

A final component of the present study shows that postnatal dexamethasone treatment significantly reduced body weight in the weanling pup. These data support similar findings reported in other experimental studies $(15,16)$ and in premature human infants (3). The deleterious effects on weight gain of dexamethasone are due to direct effects of glucocorticoids on tissue accretion and catabolism (35), explaining the lack of any protective effect on growth of co-administration with statins.

In conclusion, postnatal dexamethasone treatment in human clinically relevant doses reduced regional brain volumes, neuronal soma volume in the hippocampus and increased the density of GFAP-positive astrocytes in the white matter of the developing brain. Combined postnatal treatment of dexamethasone with pravastatin prevented the adverse effects of dexamethasone on the developing brain. The data suggest that combined glucocorticoid and statin therapy may be safer in the preterm infant than glucocorticoid treatment alone.

\section{METHODS}

\section{Ethical Approval}

The study was approved by the Cambridge University Ethical Review Committee. All procedures were carried out under the UK Animals (Scientific Procedures) 1986 Act and conducted under the authority of the appropriate project license.

\section{Animals and Experimental Design}

Fifty-three pregnant Wistar rats (Charles River, Margate, UK) with timed gestations were individually housed $\left(23 \pm 1^{\circ} \mathrm{C}\right.$, light:dark, $\left.12: 12 \mathrm{~h}\right)$ with access to food (Special Diet Services, Essex, UK) and water. All dams delivered on day 22 of gestation, P0. Litters were divided into four groups: C, $n=13$; D, $n=13$; DP, $n=15$, and CP, $n=12$.

Within $3-5 \mathrm{~h}$ of birth, pups were sexed by measurement of anogenital distance and weighed, and litters reduced to eight pups per dam (four males and four females) to standardize postnatal nutrition and maternal care. To account for sex differences, only male pups within each litter received treatment $(\mathrm{C}, n=52 ; \mathrm{D}, n=52$; DP, $n=$ 60 ; and $\mathrm{CP}, n=48$ ). C pups received injections of saline from P1 to P6. D pups received a tapering course of dexamethasone (dexamethasone-21-phosphate, disodium salt, Sigma-Aldrich, Dorset, UK; 0.5, 0.3 , and $0.1 \mu \mathrm{g} \mathrm{g}^{-1} \mathrm{day}^{-1}$ ) from P1 to P3 and saline from P1 to P6. DP pups received the same tapering course of dexamethasone from $\mathrm{P} 1$ to P3 with additional pravastatin (Sigma, Dorset, UK; $10 \mathrm{mg} \mathrm{kg}^{-1}$ ) from P1 to P6. CP pups received pravastatin from P1 to P6. Statin treatment was extended for an extra $3 \mathrm{~d}$ post glucocorticoid administration to counteract any possible lag in the effects of dexamethasone. The volume of each injection was $10 \mu \mathrm{g} \mathrm{g}^{-1}$. The treatment protocol for dexamethasone was proportional to a $21 \mathrm{~d}$ tapering course of dexamethasone used in human preterm infants to prevent or diminish chronic lung disease (16). The dose of pravastatin represents an 
intermediate level between clinical studies and doses used in previous animal experiments $(36,37)$. Body weight was recorded from P0 to P7 and every other day thereafter.

\section{Tissue Collection and Histological Analysis}

At P21, some of the pups from each litter (C: $n=8$; D: $n=7$; DP: $n=$ 8; CP: $n=7)$ were deeply anaesthetized $(0.2 \mathrm{ml}$ total volume, i.p., 100 $\mathrm{mg} \mathrm{m}^{-1}$ ketamine (Fort Dodge Animal Health, Southampton, UK) and $20 \mathrm{mg} \mathrm{ml}^{-1}$ xylazine (Millpledge Veterinary, Nottinghamshire, $\mathrm{UK})$ ) and perfused intracardially with $\mathrm{NaCl}$ followed by $4 \%$ paraformaldehyde.

The cerebrum was exhaustively sectioned at $50 \mu \mathrm{m}$ (RM 2235 vibratome, Leica Microsystems, Wetzlar, Germany). Quantification of the brain tissue was performed with an Olympus BX-50 microscope and CAST grid (Olympus, Southend-on-Sea, UK), with the observer being blind to the treatment group. Sections were washed for $30 \mathrm{~min}$ in phosphate buffered saline (PBS, Sigma-Aldrich, Dorset, UK), incubated with $30 \% \mathrm{H}_{2} \mathrm{O}_{2}$ for $5 \mathrm{~min}$ to block endogenous peroxidase activity and washed in PBS for $30 \mathrm{~min}$. Subsequently nonspecific binding was blocked with $4 \%$ bovine serum albumin (BSA) in PBS for $10 \mathrm{~min}$. Sections were then incubated with primary antibody (myelin basic protein (MBP); 1:400, Millipore, Watford, UK; neuronal nuclei antigen (NeuN), 1:400, Millipore, Watford, UK; GFAP 1:500, Millipore, Watford, UK) in primary diluent (2\% BSA in PBS containing 0.3\% Triton; Sigma-Aldrich, Dorset, UK) overnight. The following day sections were washed $30 \mathrm{~min}$ in PBS, incubated for $1 \mathrm{~h}$ with secondary antibody (1:400, Vector Laboratories, Burlingame, CA) in secondary diluent (2\% BSA in PBS) then washed for $15 \mathrm{~min}$ in PBS. Sections were incubated for $1 \mathrm{~h}$ in avidin-biotin (AB; Vector Laboratories, Peterborough, UK) in PBS and then washed in PBS for $15 \mathrm{~min}$. Staining was visualized by adding metal DAB (Thermo Scientific, Rockford, IL) in peroxide buffer (Thermo Scientific) for $2 \mathrm{~min}$ to the sections. Tissue was then mounted with $0.5 \%$ gelatin in PBS on slides. For lectin staining, the same procedure was followed as for the immunohistochemistry methods, except that the secondary antibody was omitted (1:200, Vector Laboratories, Burlingame, CA).

\section{Volumetric Analysis}

Systematic random sampling (38) was used to select, without bias, 10 sections per animal for analysis. Selected sections were stained using $1 \%$ cresyl violet. A point grid was superimposed on the sections and viewed using a $\times 1.25$ objective to assess the volume of the cerebral hemispheres, cortex, white matter, and hippocampus. Points falling on each compartment were counted and the Cavalieri principle (38) was applied in order to calculate estimated volumes:

$$
V_{(\mathrm{obj})}=t \times \sum a=t \times a_{(\mathrm{p})} \times \sum P
$$

where $V_{(\text {obj) }}$ represents the estimated volume of the brain region, $t$ is the total length of the brain $(t=$ no. of sections $\times$ section thickness), $a_{(\mathrm{p})}$ is the area associated with each point and $\Sigma P$ is the sum of points for that formation.

\section{Neuronal Number and Soma Volume Quantification Using the Fractionator Method}

Neuronal nuclei antigen-stained sections containing the cortex and hippocampus (divided into the CA1, CA2/3, and dentate gyrus) were sampled. Step motors on the microscope were used to randomly sample a known fraction of the tissue. An unbiased counting frame of known dimensions was superimposed on the tissue image. Nuclei within the counting frame, or those touching the permitted lines of the counting frame, were counted. To calculate total neuronal number, the following formula was applied:

$$
\text { est } N=\Sigma n \cdot f_{1} \cdot f_{2}
$$

where $N$ is the estimated total number of nuclei, $\Sigma n$ is the sum of nuclei counted in the brain sample, $f_{1}$ is the reciprocal of the sampling fraction, and $f_{2}$ is the areal sampling fraction.
The soma volume of neurons from the cortex and hippocampus were calculated using the vertical dissector tool in the CAST grid programme (15). Soma volume was calculated using the following formula:

$$
\mathrm{Vn}=4 \pi / 3 \times I 3 n
$$

where $I$ is the intercept measurements.

\section{Myelination}

The optical density of myelin basic protein-stained fibers was measured in the corpus callosum and cortex using ImageJ (V1.38u, National Institutes of Health, Bethesda, MD). Eight fields within the corpus callosum and cortex were examined over three sections per animal. Optical density was measured as gray levels. Nonspecific background optical densities were measured at each level in a region devoid of myelin basic protein-immunostaining and these were subtracted from the corpus callosum and cortex values. The area of myelin basic protein-positive fibers in the cortex, relative to cortical size, was assessed using the same software.

\section{Quantification of GFAP-Positive Astrocytes}

Quantitative analysis of astrocytes was performed using GFAP. Areal density of GFAP-positive astrocytes was assessed in the white matter of the cingulum (see plate 15-36 in Paxinos and Watson (39)). Labeled cells were counted at $\times 400$ magnification over eight fields per animal (two per section); values for each animal were pooled and a mean value was calculated. A mean of means \pm SEM was calculated for each group.

\section{Volume Fraction of Blood Vessels}

The volume fraction of the parenchyma occupied by blood vessels was assessed in the cortex and hipppcampus using lectin-stained sections. A point grid was superimposed on the sections and viewed at $\times 200$ magnification, and the percentage of blood vessels assessed by counting the number of intersections in up to 25 fields in both the cortex and hippocampus.

Fields were randomly sampled in the primary and secondary motor cortex and the primary somatosensory cortex, in both hemispheres over 10-12 sections. Fields in the hippocampus were taken from the stratum lacunosum-moleculare and stratum radiatum, in both hemispheres over three sections.

\section{NOx Assay}

Plasma nitrite $\left(\mathrm{NO}_{2}^{-}\right)$and nitrate $\left(\mathrm{NO}_{3}^{-}\right)$concentrations were determined at P21 in a subgroup of the pups (C: $n=10$; D: $n=6$; DP: $n=6$; CP: $n=9$ ). Levels of NOx were measured from plasma samples using the Nitrate/Nitrite Colorimetric Assay Kit (Cayman Chemical, Ann Arbor, MI) based on the principles of the Griess color reaction (40). NOx was measured from plasma samples, which were ultra-filtrated to reduce background interference due to hemoglobin. Assay buffer, nitrate standards and $40 \mu \mathrm{l}$ of plasma samples were loaded in duplicate into a 96-well microplate with nitrate reductase and an enzyme cofactor. The plates were allowed to incubate for $60 \mathrm{~min}$ at room temperature. Before a further $10 \mathrm{~min}$ incubation, Griess reagents were added to the wells. Following this, absorbance was read at $540 \mathrm{~nm}$ (Biotek ELx800 Absorbance Microplate Reader, Potton, UK). Plasma $\mathrm{NO}_{2}{ }^{-}$ levels were measured using the same method but omitting the nitrate reductase step. Plasma $\mathrm{NO}_{3}^{-}$levels were calculated as total NOx $\mathrm{NO}_{2}^{-}$. Intra-assay variability was $2.7 \%$, whereas interassay variability was $3.4 \%$. The lower limit of detection was $0.24 \mu \mathrm{mol} / 1$.

\section{Statistical Analysis}

Data are presented as mean \pm SEM unless otherwise indicated. Data were analyzed by one-way ANOVA followed by the StudentNewman-Keuls post hoc test. SigmaStat software (SigmaStat for Windows, Version 2.0) was used for all statistical analyses. $P$ values $<0.05$ were accepted as statistically significant.

\section{STATEMENT OF FINANCIAL SUPPORT}

This study was supported by the British Heart Foundation, the Biotechnology and Biological Sciences Research Council, and the Fonds Internationalisering, University Medical Center, Utrecht, The Netherlands. 
Disclosure: The authors declare no conflict of interest.

\section{REFERENCES}

1. Kinsella JP, Greenough A, Abman SH. Bronchopulmonary dysplasia. Lancet 2006;367:1421-31.

2. Halliday HL, Ehrenkranz RA, Doyle LW. Early ( $<8$ days) postnatal corticosteroids for preventing chronic lung disease in preterm infants. Cochrane Database Syst Rev 2009;CD001146.

3. Thorp JA, Jones PG, Peabody JL, Knox E, Clark RH. Effect of antenatal and postnatal corticosteroid therapy on weight gain and head circumference growth in the nursery. Obstet Gynecol 2002;99:109-15.

4. Yeh TF, Lin YJ, Lin HC, et al. Outcomes at school age after postnatal dexamethasone therapy for lung disease of prematurity. N Engl J Med 2004;350:1304-13.

5. Barrington KJ. The adverse neuro-developmental effects of postnatal steroids in the preterm infant: a systematic review of RCTs. BMC Pediatr 2001;1:1.

6. Antonow-Schlorke I, Schwab M, Li C, Nathanielsz PW. Glucocorticoid exposure at the dose used clinically alters cytoskeletal proteins and presynaptic terminals in the fetal baboon brain. J Physiol (Lond) 2003;547(Pt 1):117-23.

7. Weichsel ME Jr. The therapeutic use of glucocorticoid hormones in the perinatal period: potential neurological hazards. Ann Neurol 1977;2:3646 .

8. Kanagawa T, Tomimatsu T, Hayashi S, et al. The effects of repeated corticosteroid administration on the neurogenesis in the neonatal rat. Am J Obstet Gynecol 2006;194:231-8.

9. Iuchi T, Akaike M, Mitsui T, et al. Glucocorticoid excess induces superoxide production in vascular endothelial cells and elicits vascular endothelial dysfunction. Circ Res 2003;92:81-7.

10. Herrera EA, Verkerk MM, Derks JB, Giussani DA. Antioxidant treatment alters peripheral vascular dysfunction induced by postnatal glucocorticoid therapy in rats. PLoS ONE 2010;5:e9250.

11. Steinberg D. The statins in preventive cardiology. N Engl J Med 2008;359:1426-7.

12. Chen J, Zhang ZG, Li Y, et al. Statins induce angiogenesis, neurogenesis, and synaptogenesis after stroke. Ann Neurol 2003;53:743-51.

13. Wolozin B, Wang SW, Li NC, Lee A, Lee TA, Kazis LE. Simvastatin is associated with a reduced incidence of dementia and Parkinson's disease. BMC Med 2007;5:20.

14. Tong XK, Lecrux C, Rosa-Neto P, Hamel E. Age-dependent rescue by simvastatin of Alzheimer's disease cerebrovascular and memory deficits. J Neurosci 2012;32:4705-15.

15. Camm EJ, Tijsseling D, Richter HG, et al. Oxidative stress in the developing brain: effects of postnatal glucocorticoid therapy and antioxidants in the rat. PLoS ONE 2011;6:e21142.

16. de Vries WB, van der Leij FR, Bakker JM, et al. Alterations in adult rat heart after neonatal dexamethasone therapy. Pediatr Res 2002;52:900-6.

17. Monie IW. Comparative development of the nervous, respiratory, and cardiovascular systems. Environ Health Perspect 1976;18:55-60.

18. Watson RE, Desesso JM, Hurtt ME, Cappon GD. Postnatal growth and morphological development of the brain: a species comparison. Birth Defects Res B Dev Reprod Toxicol 2006;77:471-84.

19. Crochemore C, $\mathrm{Lu}$ J, Wu Y, et al. Direct targeting of hippocampal neurons for apoptosis by glucocorticoids is reversible by mineralocorticoid receptor activation. Mol Psychiatry 2005;10:790-8.

20. Gould E, McEwen BS, Tanapat P, Galea LA, Fuchs E. Neurogenesis in the dentate gyrus of the adult tree shrew is regulated by psychosocial stress and NMDA receptor activation. J Neurosci 1997;17:2492-8.
21. Hellsten J, Wennström M, Mohapel P, Ekdahl CT, Bengzon J, Tingström A. Electroconvulsive seizures increase hippocampal neurogenesis after chronic corticosterone treatment. Eur J Neurosci 2002;16: 283-90.

22. Kreider ML, Tate CA, Cousins MM, Oliver CA, Seidler FJ, Slotkin TA. Lasting effects of developmental dexamethasone treatment on neural cell number and size, synaptic activity, and cell signaling: critical periods of vulnerability, dose-effect relationships, regional targets, and sex selectivity. Neuropsychopharmacology 2006;31:12-35.

23. Eysel UT, Wolfhard U. The effects of partial retinal lesions on activity and size of cells in the dorsal lateral geniculate nucleus. J Comp Neurol 1984;229:301-9.

24. Mountjoy CQ, Roth M, Evans NJ, Evans HM. Cortical neuronal counts in normal elderly controls and demented patients. Neurobiol Aging 1983;4:1-11.

25. Kiernan JA, Hudson AJ. Changes in sizes of cortical and lower motor neurons in amyotrophic lateral sclerosis. Brain 1991;114 (Pt 2):843-53.

26. Volpe JJ. Neurobiology of periventricular leukomalacia in the premature infant. Pediatr Res 2001;50:553-62.

27. Gelosa P, Cimino M, Pignieri A, Tremoli E, Guerrini U, Sironi L. The role of HMG-CoA reductase inhibition in endothelial dysfunction and inflammation. Vasc Health Risk Manag 2007;3:567-77.

28. Simmons WW, Ungureanu-Longrois D, Smith GK, Smith TW, Kelly RA. Glucocorticoids regulate inducible nitric oxide synthase by inhibiting tetrahydrobiopterin synthesis and L-arginine transport. J Biol Chem 1996;271:23928-37.

29. Löhle M, Müller T, Wicher C, et al. Betamethasone effects on fetal sheep cerebral blood flow are not dependent on maturation of cerebrovascular system and pituitary-adrenal axis. J Physiol (Lond) 2005;564(Pt 2): $575-88$.

30. Ciani E, Virgili M, Contestabile A. Akt pathway mediates a cGMP-dependent survival role of nitric oxide in cerebellar granule neurones. J Neurochem 2002;81:218-28.

31. Estrada C, Murillo-Carretero M. Nitric oxide and adult neurogenesis in health and disease. Neuroscientist 2005;11:294-307.

32. Kureishi Y, Luo Z, Shiojima I, et al. The HMG-CoA reductase inhibitor simvastatin activates the protein kinase Akt and promotes angiogenesis in normocholesterolemic animals. Nat Med 2000;6:1004-10.

33. Yamashita T, Kawashima S, Ohashi Y, et al. Mechanisms of reduced nitric oxide/cGMP-mediated vasorelaxation in transgenic mice overexpressing endothelial nitric oxide synthase. Hypertension 2000;36:97-102.

34. Rybalkin SD, Yan C, Bornfeldt KE, Beavo JA. Cyclic GMP phosphodiesterases and regulation of smooth muscle function. Circ Res 2003;93:280-91.

35. Fowden AL, Forhead AJ. Endocrine mechanisms of intrauterine programming. Reproduction 2004;127:515-26.

36. Mondo CK, Yang WS, Zhang N, Huang TG. Anti-oxidant effects of atorvastatin in dexamethasone-induced hypertension in the rat. Clin Exp Pharmacol Physiol 2006;33:1029-34.

37. Torrens C, Kelsall CJ, Hopkins LA, Anthony FW, Curzen NP, Hanson MA. Atorvastatin restores endothelial function in offspring of proteinrestricted rats in a cholesterol-independent manner. Hypertension 2009;53:661-7.

38. Gundersen HJ, Jensen EB. The efficiency of systematic sampling in stereology and its prediction. J Microsc 1987;147(Pt 3):229-63.

39. Paxinos G, Watson C. The Rat Brain in Stereotaxic Coordinates. Amsterdam, The Netherlands: Elsevier, 2007.

40. Green LC, Wagner DA, Glogowski J, Skipper PL, Wishnok JS, Tannenbaum SR. Analysis of nitrate, nitrite, and [15N]nitrate in biological fluids. Anal Biochem 1982;126:131-8. 